International Mathematical Forum, 2, 2007, no. 27, 1345 - 1353

\title{
On a Decomposition of the Second Cohomology of an Abelian Topological Group
}

\author{
H. Sahleh \\ Department of Mathematics \\ Guilan University \\ P.O. Box 1914, Rasht, Iran \\ sahleh@guilan.ac.ir
}

\begin{abstract}
Let $C$ be an abelian topological group , $A$ a trivial $C$-module.In this paper we show that the second cohomology of $C$ with the 2-divisible coefficient is isomorphic to the direct sum of the second cohomology of a Lie ring $c$, whose underlying group is $C$, and the group of symmetric cocycles. Also there is an isomorphism between the categories of abelian topological groups of nilpotency class two,with a continuous section , and that of Lie rings of nilpotency class two.
\end{abstract}

Mathematics Subject Classification: 22A05

Keywords: Topological extension ; Second cohomology; Lie ring

\section{Introduction}

All spaces are assumed to be Tychanov (completely regular, Hausdorff).A topological extension of $G$ by $N$ is a short exact sequence $0 \rightarrow N \stackrel{i}{\rightarrow} Q \stackrel{\pi}{\rightarrow} G \rightarrow$ 0 , where $i$ is a topological embedding onto a closed subgroup and $\pi$ an open continuous onto homomorphism. The extension is central if $N$ is in the center of $G$. We consider extensions with a continuous section i.e. $u: G \rightarrow Q$ such that $\pi u=I d$. For example, if $G$ is a connected locally compact group ,then any topological extension of $G$ by a connected simply connected Lie group has a continuous section [6,theorem 2]. Notation and definitions as in [2].

In section 1 , we give some results concerning the abelian group of nilpotency class two. In section 2 , an isomorphism is constructed between the second cohomology of an abelian topological group $C$ and the direct sum of the second cohomology of a Lie ring $c$, with the underlying group $C$, and the group of symmetric cocycles with the trivial coefficient. 


\section{Nilpotent group of class two}

In this section we define nilpotent topological group of class two and express it as an exact sequence with a continuous section. Moreover, we define an operation on a nilpotent group $G$ of class two and will show that it induces the same group structure on $G$.

Definition 1.1 Let $G$ be an abelian topological group .Then $G$ is called a nilpotent group of class two if there exists an extension ,(e): $0 \rightarrow A \rightarrow G \rightarrow$ $C \rightarrow 0$, with a continuous section where $A$ and $C$ are abelian and $A$ is in the center of $G$.

\section{Cohomology of topological groups}

Let $G$ be a topological group and $A$ an abelian topological group on which $G$ acts continuously.

Let $C^{n}(G, A)$ be the continuous maps $\phi: G^{n} \rightarrow A$ with the coboundary map

$$
\left.C^{n}(G, A) \stackrel{\delta_{n}}{\rightarrow} C^{n+1}(G, A)\right)
$$

given by

$$
\begin{aligned}
\delta \phi\left(g_{1}, \ldots, g_{n}\right)= & g_{1} . \phi\left(g_{2}, \ldots, g_{n}\right) \\
& +\sum_{i=1}^{n-1}(-1)^{i} \phi\left(g_{1}, \ldots, g_{i} g_{i+1}, \ldots, g_{n}\right)+(-1)^{n} \phi\left(g_{1}, \ldots, g_{n-1}\right)
\end{aligned}
$$

Note that this is analogous to the inhomogeneous resolution for the discrete case.

Definition1.2 .The continuous group cohomology of $G$ with coefficient in $A$ is

$$
H^{n}(G, A)=\operatorname{Ker} \delta_{n} / \operatorname{Im} \delta_{n-1}
$$

Let $\operatorname{Ext}_{s}(C, A)$ be the set of extensions of $A$ by $C$ with a continuous section. It is known [1] ,by the Baer sum, that $\operatorname{Ext}_{s}(C, A)$ is an abelian topological group. By [3], if $C$ is a topological group and $A$ a trivial $C$-module then there is an isomorphism between the second cohomology of $C$ and the group of extensions of $C$ by $A$ with continuous sections, namely

$$
H^{2}(C, A) \simeq \operatorname{Ext}_{s}(C, A)
$$

Let $G$ be a nilpotent group of class two and (e) : $0 \rightarrow A \rightarrow G \rightarrow C \rightarrow 0$ the corresponding extension. In fact, $G$ and $A \times C$ are homeomorphic [1]. Now we define a new operation on $G$ and will show that this is a group structure.

By [3], there is a 2-cocycle $[\phi] \in H^{2}(C, A)$ which corresponds to (e).

$$
\left(a_{1}, c_{1}\right) \cdot\left(a_{2}, c_{2}\right)=\left(a_{1}+a_{2}+\phi\left(c_{1}, c_{2}\right), c_{1}+c_{2}\right), \quad a_{1}, a_{2} \in A, \quad c_{1}, c_{2} \in C
$$


By the cocycle condition it is easy to show that the operation is associative. Since $[\phi] \in H^{2}(C, A)$ and $A$ is a trivial module, the associativity implies the following identity:

$$
\phi\left(c_{1}, c_{2}\right)+\phi\left(c_{1}+c_{2}, c_{3}\right)=\phi\left(c_{1}, c_{2}+c_{3}\right)+\phi\left(c_{2}, c_{3}\right)
$$

Hence,$\phi(0, c)=\phi(c, 0)=\phi(0,0)$. For such $\phi$, then $(-\phi(0,0), 0)$ is the identity of $G$ :

$$
(a, c)(-\phi(0,0), 0)=(a-\phi(0,0), 0)+\phi(c, 0), c)=(a, c)
$$

Now for the inverse element set $(a, c)^{-1}=(-a-\phi(c,-c)-\phi(0,0), c)$.

The following lemma indicates that the group structure of a nilpotent topological group of class two is uniquely determined by 2-cocycles modulo coboundaries of 1-chains.

Lemma 1.3 Let $G$ be a nilpotent topological group of class two and $0 \rightarrow$ $A \rightarrow G \rightarrow C \rightarrow 0$ the associated extension of $G$. Then the group structure of $G$ is uniquely determined by the elements of $H^{2}(C, A)$, with $A$ a trivial module.

Proof: Let $G$ be a nilpotent group of class two and $0 \rightarrow A \rightarrow G \rightarrow C \rightarrow 0$ the central extension where $A$ and $C$ are abelian . Let $\phi \in Z^{2}(C, A)$ be the associated 2-cocycle of the extension.In this case ,the maps

$$
A \rightarrow A \times\{0\}, a \mapsto(a-\phi(0,0), 0)
$$

and

$$
C \rightarrow G / A, c \mapsto(0, c) A
$$

are isomorphisms.

Now we choose other representation than $(0, c)$. For any continuous function $q: C \rightarrow A$, define $C \rightarrow G / A$

$$
c \mapsto(0, c)(q(c)-\phi(0,0), 0) A=(q(c), c) A
$$

The map $\psi: G \rightarrow G,(a, c) \mapsto(a+q(c), c)$ is one-one ,onto continuous homomorphism:

$$
\begin{aligned}
\psi\left(\left(a_{1}, c_{1}\right) \cdot\left(a_{2}, c_{2}\right)\right) & =\left(a_{1}+a_{2}+\phi\left(c_{1}, c_{2}\right)+q\left(c_{1}+c_{2}\right), c_{1}+c_{2}\right) \\
& =\left(a+1+a_{2}+\phi\left(c_{1}, c_{2}\right)+q\left(c_{1}\right)+q\left(c_{2}\right), c_{1}+c_{2}\right) \\
& =\psi\left(\left(a_{1}, c_{1}\right)\right) \phi\left(\left(a_{2}, c_{2}\right)\right)
\end{aligned}
$$

Now if we consider $(a+q(c), c)$ as an element of $G$ we conclude that the same group structure in $G$ can be defined by 2-cocycle $\phi\left(c_{1}, c_{2}\right)+q\left(c_{1}\right)+q\left(c_{2}\right)-$ 
$q\left(c_{1}+c_{2}\right)$. Note that the difference between $\phi$ and this new 2-cocycle is equal to the coboundaries of 1-chain $q$. Hence The group structure in $G$ is uniquely determined by 2-cocycle modulo coboundaries of 1-chains, that is, by $H^{2}(C, A)$.

Definition 1.4 A 2-cocycle $\psi \in C^{2}(C, A)$ is called central iff $\psi(0,0)=0$.

If $\psi$ is central then for any $c \in C$

$$
\psi(0, c)=\psi(c, 0)=\psi(0,0)=0
$$

By choosing $q(0)=-\psi(0,0)$, we get a central cocycle.

Definition 1.5 An abelian topological group is 2-divisible if the continuous homomorphism $A \rightarrow A, a \mapsto a+a$ is an automorphism.

For example an abelian topological p-group is 2 -divisible [4]. Let $a / 2$ be the image of $a$ under the inverse map.

Definition 1.6 A cocycle $\psi \in C^{2}(C, A)$ is normalized iff $\psi(c,-c)=0$ for all $c \in C$.

If $\psi$ is normalized then $(a, c)^{-1}=(-a,-c)$ for any $(a, c) \in G$

Lemma 1.7 For a 2-divisible abelian topological group $A$ and an abelian group $C$, every element of $H^{2}(C, A)$ can be represented by a normalized cocycle.

proof : if $A$ is a 2-divisible abelian group and $\psi$ is a central cocycle,then $q(c)=\psi(c,-c) / 2$ gives a normalized cocycle.

Remark 1.8. Note that by either of the following conditions :

(1) $q(0)=0$ for central cocycle ,

(2) $q(-c)=-q(c)$ for all $c$ and normalized cocycles,

if the coboundary of a 1-chain $q$ is added to a central (normalized )cocycle,then the result is still central (normalized).

\section{$2 \quad$ Lie Ring}

In this section we define the topological Lie ring of nilpotentecy class two and will find similar results as in the previous part ( for more on Lie ring see [5]). 
Definition 2.1 A topological Lie ring means an abelian topological group with the following conditions:

(1) the commutator [,] is bilinear

(2) $[a, a]=0 \Leftrightarrow[a, b]=-[b, a]$, foralla, $b$

(3) $[[a, b], c]+[[b, c], a]+[[c, a], b]=0$

As usual we denote the commutator $a b-b a$ of elements $a, b$ in the lie ring by $[a, b]$. A commutative Lie ring means zero commutator.

Definition 2.2 A topological Lie ring $n$ is of nilpotency class two if there exists a central extension of Lie rings

$$
0 \rightarrow a \rightarrow n \rightarrow c \rightarrow 0
$$

with a continuous section $c \rightarrow n$, where $a$ and $c$ are commutative Lie rings.

Note that the extension corresponds to a 2-cocycle $\phi \in C^{2}(C, A)$ and every element of $n$ can be written as $n=(a, c), a \in a, c \in c$. Now we define a new operation on $n$ :

$$
\left(a_{1}, c_{1}\right) \cdot\left(a_{2}, c_{2}\right)=\left(a_{1}+a_{2}+\phi\left(c_{1}, c_{2}\right), c_{1}+c_{2}\right), a_{1}, a_{2} \in A, c_{1}, c_{2} \in C
$$

and

$$
\left.\left[\left(a_{1}, c_{1}\right), a_{2}, c_{2}\right)\right]=\left[\eta\left(c_{1}, c_{2}\right)-\phi(0,0), 0\right]
$$

where $\eta\left(c_{1}, c_{2}\right)=\phi\left(c_{1}, c_{2}\right)-\phi\left(c_{2}, c_{1}\right)$.

The commutator properties are equivalent to the fact that $\eta: C \times C \rightarrow A$ is a skew-symmetric bihomomorphism, that is,$\eta\left(c_{1}, c_{2}\right)=-\eta\left(c_{2}, c_{1}\right)$. This implies that $\eta(0,0)=0$. In other word $\eta$ is a 2-cocycle,$\eta \in C^{2}(c, A)$ with the trivial action of $c$ on $A$.

In this construction

$$
a \rightarrow a \times\{c\}, a \mapsto(a-\phi(0,0), 0)
$$

and

$$
c \rightarrow n / a, c \mapsto(0, c)+a
$$

are Lie ring isomorphisms.

If we choose another representation of coset than $(0, c)$ for any function $q$ : $C \rightarrow A$

$$
c \rightarrow n / a, c \mapsto(0, c)+(q(c)-\phi(0,0))+a=(q(c), c)+a
$$

From (2.2) we get the same Lie ring structure in $n$. The same Lie ring structure in $n$ can be defined by 2-cocycle :

$$
\phi\left(c_{1}, c_{2}\right)+q\left(c_{2}\right)-q\left(c_{1}+c_{2}\right)
$$


obtaining from $\phi$ by adding a 1-chain. Note that the coboundary of a 1-chain $r \in C^{1}(c, A)$ with the trivial action of $c$ on $A$, is $r\left(c_{1}, c_{2}\right)=r\left[c_{1}, c_{2}\right]=0$. Thus, the Lie ring structure in $n$ is uniquely determined by elements of $H_{\text {sym }}^{2}(C, A) \oplus$ $H^{2}(c, A)$ with the trivial actions of $C$ and $c$ on $A$, where $H_{\text {sym }}^{2}(C, A)$ denotes the subgroup of second cohomology classes defined by symmetric cocycles. Again note that we need the central extensions in order the cocycles be nondegenerate.

Definition 2.3 A skew-symmetric bihomomorphism $\eta: C \times C \rightarrow A$ is non-degenerate iff for any $c_{1} \neq 0$, there exists $c_{2} \in C$ such that $\eta\left(c_{1}, c_{2}\right) \neq 0$

For a non-degenerate cocycle $\eta$, element $\left(a_{1}, a_{2}\right) \in n$ with $c_{1} \neq 0$ can not be central since its commutator with $\left(0, c_{2}\right)$ is not $0,\left(c_{2}\right.$ as in definition 2.3).

In the next result $C_{\text {sym }}^{2}$ will be the symmetric cocycles and $H_{\text {sym }}^{2}(C, A)$ the subgroup of 2-cocycles defined by symmetric cocycles.

Theorem 2.4 Let $A$ be a 2-divisible abelian topological group and $C$ an abelian topological group and $c$ a commutative Lie ring with underlying abelian group of which is $C$. Let $c$ and $C$ act trivially on $A$.Then

$$
L_{c}: C^{2}(C, A) \rightarrow C_{\text {sym }}^{2}(C, A) \oplus C^{2}(c, A), \quad \psi \mapsto(\phi, \eta)
$$

is an isomorphism, factor of which by coboundaries of 1-chain is an isomorphism

$$
L_{h}: H^{2}(C, A) \rightarrow H_{s y m}^{2}(C, A) \oplus H^{2}(c, A)
$$

Proof. Consider the following map:

$$
\begin{aligned}
L_{c}: C^{2}(C, A) & \rightarrow C_{s y m}^{2}(C, A) \oplus C^{2}(c, A) \\
\psi & \mapsto(\phi, \eta)
\end{aligned}
$$

where

$$
\begin{aligned}
& \text { (2.4.1) } \quad \phi\left(c_{1}, c_{2}\right)=\frac{\psi\left(c_{1}, c_{2}\right)+\psi\left(c_{2}, c_{1}\right)}{2} \\
& (2.4 .2) \quad \eta\left(c_{1}, c_{2}\right)=\psi\left(c_{1}, c_{2}\right)+\psi\left(c_{2}, c_{1}\right)
\end{aligned}
$$

Note that $\phi$ is a cocycle and symmetric,

$$
\phi\left(c_{1}, c_{2}\right)=\phi\left(c_{2}, c_{1}\right)
$$

Also $\eta$ is Skew-symmetric.

Now we show that $\eta$ is a bihomomorphism. By the cocycle identities :

$$
\psi\left(c_{1}+c_{2}, c_{3}\right)+\psi\left(c_{1}, c_{2}\right)=\psi\left(c_{1}, c_{2}+c_{3}\right)+\psi\left(c_{2}, c_{3}\right)
$$




$$
\begin{gathered}
\psi\left(c_{3}+c_{2}, c_{1}\right)+\psi\left(c_{3}, c_{1}\right)=\psi\left(c_{3}, c_{1}+c_{2}\right)+\psi\left(c_{1}, c_{2}\right) \\
-\psi\left(c_{1}+c_{3}, c_{2}\right)-\psi\left(c_{1}, c_{3}\right)=-\psi\left(c_{1}, c_{3}+c_{2}\right)-\psi\left(c_{3}, c_{2}\right)
\end{gathered}
$$

Adding these, we get

$\psi\left(c_{1}+c_{2}, c_{3}\right)+\psi\left(c_{3}, c_{1}\right)-\psi\left(c_{1}, c_{3}\right)=\psi\left(c_{3}, c_{1}+c_{2}\right)+\psi\left(c_{2}, c_{3}\right)-\psi\left(c_{3}, c_{2}\right)$

Hence, $\eta\left(c_{1}+c_{2}, c_{3}\right)=\eta\left(c_{1}, c_{3}\right)+\eta\left(c_{2}, c_{3}\right)$

The map $L_{c}$, by linearity, is a continuous homomorphism . Now $L_{c}$ induces a continuous homomorphism $L_{h}: H^{2}(C, A) \rightarrow H_{\text {sym }}^{2}(C, A) \oplus H^{2}(c, A)$; since adding a coboundary to $\psi$ adds the same coboundary to $\phi$ and $H^{2}(c, A)=$ $C^{2}(c, A)$. On the other hand, we define

$$
\begin{aligned}
& E_{c}: C_{\text {sym }}^{2}(C, A) \oplus C^{2}(c, A) \rightarrow C^{2}(C, A) \\
& (2.4 .3) \quad \psi\left(c_{1}, c_{2}\right)=\phi\left(c_{1}, c_{2}\right)+\frac{\eta\left(c_{1}, c_{2}\right)}{2}
\end{aligned}
$$

Since every bihomomorphism from $C \times C$ to $A$ is an element of $C^{2}(C, A)$ so $\eta$ is a cocycle and by linearity $\phi$ is a cocycle. Similarly, $E_{c}$ is a continuous homomorphism and induces

$$
E_{h}: H_{\text {sym }}^{2}(C, A) \oplus H^{2}(c, A) \rightarrow H^{2}(C, A)
$$

It is easy to show that $L_{h} \circ E_{h}=I d$ and vice versa. Hence $L_{h}$ is an isomorphism.

Remark 2.5.

(1) Let $G$ be an abelian topological group of nilpotency class two with a 2-divisible center and $L(G)$ the Lie ring of nilpotency class two with 2divisible center defined on the underlying set of $G$ by cocycles (2.4.1), (2.42)..By Theorem 2.3 this construction does not depend on the choice of the cocycle defining a group structure in $G$.

(2) For a Lie ring $n$ of nilpotency class two with 2-divisible center, denote $E(n)$ the group of nilpotency class two the underlying set $n$ by a cocycle $(2.4 .3)$

By theorem 2.4, this construction does not depend on the choice of the cocycles $\phi, \eta$ defining a Lie ring structure in $n$. Denote the categories of abelian topological groups of nilpotency class two with 2-divisible center and Lie rings of nilpotency class two with 2 -divisible center by $\mathcal{G}_{*}, \mathcal{L}_{*}$ respectively.

Theorem 2.6 There is an isomorphism between $\mathcal{G}_{*}, \mathcal{L}_{*}$. 
Proof. Let $G_{1}, G_{2} \in \mathcal{G}_{*}, f: G_{1} \rightarrow G_{2}$ be a continuous homomorphism and consider $f$ as a map from $L\left(G_{1}\right)$ to $L\left(G_{2}\right)$ and denote it by $L(f)$. Similarly, for a continuous homomorphism $f: g_{1} \rightarrow g_{2}, E(f)$ is $f$ considered as a map from $E\left(n_{1}\right)$ to $E\left(n_{2}\right)$.

Note that by lemma 1.7 for a 2-divisible abelian topological group $A$, and an abelian group $C$ every element of $H^{2}(C, A)$ can be represented by a normalized cocycle. Choose cocycles $\psi_{1}, \psi_{2}$ so that they define the group structure on $G_{1}$ and $G_{2}$,respectively. So

$$
\begin{aligned}
& \phi_{1}\left(c_{1}, c_{2}\right)=\frac{\psi_{1}\left(c_{1}, c_{2}\right)+\psi_{1}\left(c_{2}, c_{1}\right)}{2} \\
& \phi_{2}\left(c_{1}, c_{2}\right)=\frac{\psi_{2}\left(c_{1}, c_{2}\right)+\psi_{2}\left(c_{2}, c_{1}\right)}{2}
\end{aligned}
$$

are normalized. Now we show that if $f$ is a group homomorphism, then $L(f)$ is a Lie ring homomorphism. Consider the following identities:

$$
\begin{aligned}
f\left(a_{1}+a_{2}+\psi_{1}\left(c_{1}, c_{2}\right), c_{1}+c_{2}\right)= & f\left(a_{1}, c_{1}\right) \cdot f\left(a_{2}, c_{2}\right) \\
= & \left(a_{1}, \gamma_{1}\right) \cdot\left(a_{2}, \gamma_{2}\right) \\
= & \left(a_{1}+a_{2}+\psi_{2}\left(\gamma_{1}, \gamma_{2}\right)\right. \\
f\left(-a_{1}-a_{2}-\psi_{1}\left(c_{1}, c_{2}\right),-c_{1}-c_{2}\right) & =\left(f\left(a_{2}, c_{2}\right) \cdot f\left(a_{1}, c_{1}\right)\right)^{-1} \\
& =\left(\left(a_{2}, \gamma_{2}\right) \cdot\left(a_{1}, \gamma_{1}\right)\right)^{-1} \\
& =\left(-a_{1}-a_{2}-\psi_{2}\left(\gamma_{1}, \gamma_{2}\right),-\gamma_{1}-\gamma_{2}\right)
\end{aligned}
$$

Multiplying the left and the right hand sides, we get

$$
f\left(\eta_{1}\left(c_{1}, c_{2}\right), 0\right)=\left(\eta_{2}\left(\gamma_{1}, \gamma_{2}\right), 0\right)
$$

Now dividing the central element by 2 and inversion we get,

$$
f\left(-\frac{\eta_{1}\left(c_{1}, c_{2}\right)}{2}, 0\right)=\left(-\frac{\eta_{2}\left(\gamma_{1}, \gamma_{2}\right)}{2}, 0\right)
$$

So

$$
f\left(a_{1}+a_{2}+\phi_{1}\left(c_{1}+c_{2}\right), c_{1}+c_{2}\right)=\left(a_{1}+a_{2}+\phi_{2}\left(\gamma_{1}, \gamma_{2}\right), \gamma_{1}+\gamma_{2}\right)
$$

Hence

$$
L(f)\left(n_{1}+n_{2}\right)=L(f) n_{1}+L(f) n_{2} \quad n_{1}=\left(a_{1}, c_{1}\right), \quad n_{2}\left(a_{2}, c_{2}\right)
$$

This formula together with (2.5.1) gives

$$
L(f)\left(\left[n_{1}, n_{2}\right]\right)=\left[L(f) n_{1}, L(f) n_{2}\right]
$$

that is $L(f)$ is a Lie ring homomorphism . Similarly for $f$ a Lie ring homomorphism,$E(f)$ is a group homomorphism. 


\section{References}

[1] R. C. Alperin,H. Sahleh, Hopf,s formula and the Schur multiplicator for topological groups, Kyungpook .Math.Journal. vol 31,no 1(1991), 35-71.

[2] E. Hewitt, K. A. Ross, Abstract Harmonic Analysis I, Academic Press, New York , 1963.

[3] S.T.Hu, Cohomology theory in topological groups,Michigan Math.J. 1(1) (1952), 11-59.

[4] A. Mihailovs, The orbite Method for Finite Groups of Nilpotency Class Two of Odd Order ,priprint

[5] J.R.Robinson, A course in the theory of groups, Springer-verlag, New York , 1982.

[6] A. Shtern , Remarks on Pseudochrscters and the Real Continuous Bounded Cohomology of Connected Locally Compact Groups, annals of Global analysis and Geometry 20 (2001),199-221

\section{Received: October 31, 2006}

